\title{
Remarks on logarithmic K-stability
}

\author{
Chi Li
}

ABSTRACT: We make some observation on the logarithmic version of K-stability.

\section{Introduction}

Let $(X, J)$ be a Fano manifold, that is, $K_{X}^{-1}$ is ample. The basic problem in Kähler geometry is to determine whether $(X, J)$ has a Kähler-Einstein metric (cf. [12])

On way to attack this problem is to use continuity method. Fix a reference Kähler metric $\omega \in c_{1}(X)$. Its Ricci curvature Ric $(\omega)$ also lies in $c_{1}(X)$. So there exists $h_{\omega} \in C^{\infty}(X)$ such that

$$
\operatorname{Ric}(\omega)-\omega=\partial \bar{\partial} h_{\omega}, \quad \int_{X} e^{h_{\omega}} \omega^{n}=\int_{X} \omega^{n}
$$

Consider the following family of Monge-Ampère equations.

$$
\left(\omega+\partial \bar{\partial} \phi_{t}\right)^{n}=e^{h_{\omega}-t \phi} \omega^{n}
$$

This is equivalent to the equation for Kähler forms:

$$
\operatorname{Ric}\left(\omega_{\phi}\right)=t \omega_{\phi}+(1-t) \omega
$$

By Yau's theorem [16], we can always solve $(*)_{t}$ for $t=0$. If we could solve $(*)_{t}$ for $t=1$, we would get Kähler-Einstein metric. However, it was first showed by Tian [13] that we may not be able to solve $(*)_{t}$ on certain Fano manifold for $t$ sufficiently close to 1 . Equivalently, for such a Fano manifold, there is some $t_{0}<1$, such that there is no Kähler metric $\omega$ in $c_{1}(X)$ which can have $\operatorname{Ric}(\omega) \geq t_{0} \omega$.

The existence problem of Kähler-Einstein metric is a special case of the existence problem of constant scalar curvature Kähler (cscK) metric. For the latter, we fix an ample line bundle $L$ on $(X, J)$. We have the following folklore conjecture. For the definition of K-stability, see [14], [3] or Definition 4.

Conjecture 1 (Tian-Yau-Donaldson). ([14],[3]) There is a smooth constant scalar curvature Kähler metric in $c_{1}(L)$ on $(X, J)$ if and only if $(X, J, L)$ is K-stable.

Return to the continuity method $(*)_{t}$ and let $R(X)=\sup \left\{t:(*)_{t}\right.$ is solvable $\}$. Székelyhidi proved that

Proposition 1 ([10]).

$$
R(X)=\sup \left\{t: \exists \text { a Kähler metric } \omega \in c_{1}(X) \text { such that Ric }(\omega)>t \omega\right\}
$$

In particular, $R(X)$ is independent of reference metric $\omega$.

There is another continuity method we can try. Let $Y \in\left|-K_{X}\right|$ be a general element, then $Y$ is a smooth Calabi-Yau hypersurface. The Kähler-Einstein metric with cone singularity along $Y$ of cone angle $2 \pi \beta$ is a solution to the following distributional equation

$$
\operatorname{Ric}(\omega)=\beta \omega+(1-\beta)\{Y\}
$$


Conjecture 2 (Donaldson). There is a cone-singularity solution $\omega_{\beta}$ to $(2)$ for any parameter $\beta \in(0, R(X))$. If $R(X)<1$, there is no solution for parameter $\beta \in(R(X), 1)$.

The purpose of this note is to discuss the logarithmic version of K-stability and prove the following result.

Theorem 1. Let $X_{\triangle}$ be a toric Fano variety with a $\left(\mathbb{C}^{*}\right)^{n}$ action. Let $Y$ be a general hyperplane section of $X_{\triangle}$. When $\beta<R\left(X_{\triangle}\right),\left(X_{\triangle}, \beta Y\right)$ is log-K-stable along any 1 parameter subgroup in $\left(\mathbb{C}^{*}\right)^{n}$. When $\beta=R\left(X_{\triangle}\right),\left(X_{\triangle}, \beta Y\right)$ is semi-log-K-stable along any 1 parameter subgroup in $\left(\mathbb{C}^{*}\right)^{n}$ and there is a 1 parameter subgroup in $\left(\mathbb{C}^{*}\right)^{n}$ which has vanishing log-Futaki invariant. When $\beta>R\left(X_{\triangle}\right),\left(X_{\triangle}, \beta Y\right)$ is not log-K-stable.

This explains and generalizes slightly the calculation in [4] and gives some evidence for the Conjecture 2 (Combined with Conjecture 3).

We prove the above result by calculating $R\left(X_{\triangle}\right)$ and log-Futaki invariant explicitly. $R\left(X_{\triangle}\right)$ was calculated in [6] based on Wang-Zhu's work [15]. The main formula for log-Futaki invariant is (19).

A toric Fano manifold $X_{\triangle}$ is determined by a reflexive lattice polytope $\triangle$ (For details on toric manifolds, see [8]). For example, let $B l_{p} \mathbb{P}^{2}$ denote the manifold obtained by blowing up one point on $\mathbb{P}^{2}$. Then $B l_{p} \mathbb{P}^{2}$ is a toric Fano manifold and is determined by the following polytope.

Any such polytope $\triangle$ contains the origin $O \in \mathbb{R}^{n}$. We denote the barycenter of $\triangle$ by $P_{c}$. If $P_{c} \neq O$, the ray $P_{c}+\mathbb{R}_{\geq 0} \cdot \overrightarrow{P_{c} O}$ intersects the boundary $\partial \triangle$ at point $Q$.

Theorem 2. [6] If $P_{c} \neq O$,

$$
R\left(X_{\triangle}\right)=\frac{|\overline{O Q}|}{\left|\overline{P_{c} Q}\right|}
$$

Here $|\overline{O Q}|,\left|\overline{P_{c} Q}\right|$ are lengths of line segments $\overline{O Q}$ and $\overline{P_{c} Q}$. In other words,

$$
Q=-\frac{R\left(X_{\triangle}\right)}{1-R\left(X_{\triangle}\right)} P_{c} \in \partial \triangle
$$

If $P_{c}=O$, then there is Kähler-Einstein metric on $X_{\triangle}$ and $R\left(X_{\triangle}\right)=1$.

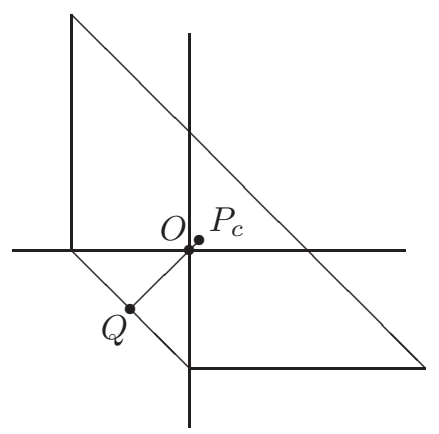

Acknowledgement: I would like to thank Professor Gang Tian for helpful discussion and constant encouragement. In particular, he suggested the log-K-stability and told me the result in [7] which provides the starting example of the logarithmic version of Tian-Yau-Donaldson conjecture. 


\section{Log-Futaki invariant}

In this section, we recall Donaldson's definition of log-Futaki invariant $(6)$. Let $(X, L)$ be a polarized projective variety and $D$ be a normal crossing divisor:

$$
D=\sum_{i=1}^{r} \alpha_{i} D_{i}
$$

with $\alpha_{i} \in(0,1)$.

From now on, we fix a Hermitian metric $\|\cdot\|_{i}=h_{i}$ and defining section $s_{i}$ of the line bundle $\left[D_{i}\right]$.

Assume $\omega \in c_{1}(L)$ is a smooth Kähler form. We define

$$
\overline{\mathcal{P}}(\omega)=\left\{\omega_{\phi}:=\omega+\frac{\sqrt{-1}}{2 \pi} \partial \bar{\partial} \phi ; \phi \in L^{\infty}(X) \cap C^{\infty}(X \backslash D) \text { such that } \omega+\frac{\sqrt{-1}}{2 \pi} \partial \bar{\partial} \phi \geq 0\right\}
$$
by

Around any point $p \in X$, we can find local coordinate $\left\{z_{i} ; i=1, \cdots, n\right\}$, such that $D$ is defined

$$
D=\cup_{i=1}^{r_{p}} \alpha_{i}\left\{z_{i}=0\right\}
$$

where $r_{p}=\sharp\left\{i ; p \in D_{i}\right\}$.

Definition 1. We say that $\hat{\omega} \in \overline{\mathcal{P}}(\omega)$ is a conic Kähler metric on $(X, D)$, if around $p$, $\omega$ is quasi-isometric to the metric

$$
\sum_{i=1}^{r_{p}} \frac{d z_{i} \wedge d \bar{z}_{i}}{\left|z_{i}\right|^{2 \alpha_{i}}}+\sum_{j=r_{p}+1}^{n} d z_{j} \wedge d \bar{z}_{j}
$$

We will simply say that $\hat{\omega}$ is a conic metric if it's clear what $D$ is.

Geometrically, this means the Riemannian metric determined by $\omega$ has conic singularity along each $D_{i}$ of conic angle $2 \pi\left(1-\alpha_{i}\right)$.

Remark 1. Construction of Kähler-Einstein metrics with conic singularites was proposed long time ago by Tian, see [11] in which he used such metrics to prove inequalities of Chern numbers in algebraic geometry.

One consequence of this definition is that globally the volume form has the form

$$
\hat{\omega}^{n}=\frac{\Omega}{\prod_{i=1}^{r}\left\|s_{i}\right\|_{i}^{2 \alpha_{i}}}
$$

where $\Omega$ is a smooth volume form. For any volume form $\Omega$, let $\operatorname{Ric}(\Omega)$ denote the curvature of the Hermitian metric on $K_{X}^{-1}$ determined by $\Omega$. Then, by abuse of notation,

$$
\begin{aligned}
\operatorname{Ric}(\hat{\omega}) & =\operatorname{Ric}\left(\hat{\omega}^{n}\right)=\operatorname{Ric}(\Omega)+\frac{\sqrt{-1}}{2 \pi} \sum_{i=1}^{r} \alpha_{i} \partial \bar{\partial} \log \left\|s_{i}\right\|_{i}^{2}=\operatorname{Ric}(\Omega)-\sum_{i=1}^{r} \alpha_{i} c_{1}\left(\left[D_{i}\right], h_{i}\right)+\sum_{i=1}^{r} \alpha_{i}\left\{D_{i}\right\} \\
& =\operatorname{Ric}(\Omega)-c_{1}([D], h)+\{D\}
\end{aligned}
$$

where $h=\otimes_{i=1}^{r} h_{i}^{\alpha_{i}}$ and $s=\otimes_{i=1}^{r} s_{i}^{\alpha_{i}}$ are Hermitian metric and defining section of the $\mathbb{R}$-line bundle $[D]=\otimes_{i=1}^{r}\left[D_{i}\right]^{\alpha_{i}}$.

Here we used the Poincáre-Lelong identity:

$$
\frac{\sqrt{-1}}{2 \pi} \partial \bar{\partial} \log \left\|s_{i}\right\|_{i}^{2}=-c_{1}\left(\left[D_{i}\right], h_{i}\right)+\left\{D_{i}\right\}
$$


where $\left\{D_{i}\right\}$ is the current of integration along the divisor $D_{i}$.

The scalar curvature of $\hat{\omega}$ on its smooth locus $X \backslash D$ is

$$
S(\hat{\omega})=\hat{g}^{i \bar{j}} \hat{R}_{i \bar{j}}=\frac{n \operatorname{Ric}(\hat{\omega}) \wedge \hat{\omega}^{n-1}}{\hat{\omega}^{n}}=\frac{n\left(\operatorname{Ric}(\Omega)-c_{1}([D], h)\right) \wedge \hat{\omega}^{n-1}}{\hat{\omega}^{n}}
$$

So if $S(\hat{\omega})$ is constant, then the constant only depends on cohomological classes by the identity:

$$
n \mu_{1}:=\frac{n\left(c_{1}(X)-c_{1}([D])\right) \wedge\left[c_{1}(L)\right]^{n-1}}{c_{1}(L)^{n}}=\frac{-n\left(K_{X}+D\right) \cdot L^{n-1}}{L^{n}}=n \mu-\frac{\operatorname{Vol}(D)}{\operatorname{Vol}(X)}
$$

Here

$$
n \mu=\frac{n c_{1}(X) \cdot c_{1}(L)^{n-1}}{c_{1}(L)^{n}}=\frac{-n K_{X} \cdot L^{n-1}}{L^{n}}
$$

is the average scalar curvature for smooth Kähler form in $c_{1}(L)$. And

$$
\operatorname{Vol}(D)=\int_{D} \frac{c_{1}(L)^{n-1}}{(n-1) !}=\frac{D \cdot L^{n-1}}{(n-1) !}, \quad \operatorname{Vol}(X)=\int_{X} \frac{c_{1}(L)^{n}}{n !}=\frac{L^{n}}{n !}
$$

Now assume $\mathbb{C}^{*}$ acts on $(X, L)$ and $v$ is the generating holomorphic vector field. Recall that the ordinary Futaki-Calabi invariant ([5], [2]) is defined by

$$
F\left(c_{1}(L)\right)(v)=-\int_{X} \theta_{v}(S(\omega)-n \mu) \frac{\omega^{n}}{n !}
$$

where $\theta_{v}$ satisfies

$$
\iota_{v} \omega=\bar{\partial} \theta_{v}
$$

Now assume $\hat{\omega}_{\infty} \in \overline{\mathcal{P}}(\omega)$ is a conic metric and satisfies

$$
S\left(\hat{\omega}_{\infty}\right)=n \mu_{1}
$$

Assume $D$ is preserved by the $\mathbb{C}^{*}$ action. Let's calculate the ordinary Futaki invariant using the conic metric $\hat{\omega}_{\infty}$. Let $\hat{\theta}_{v}=\hat{\theta}\left(\hat{\omega}_{\infty}, v\right)$. Then near $p \in D, v \sim \sum_{i=1}^{r_{p}} c_{i} z_{i} \partial_{z_{i}}+\tilde{v}$ with $\tilde{v}=o\left(z_{1} \cdots z_{r_{p}}\right)$ holomorphic. $\hat{\theta}_{v} \sim \sum_{i=1}^{r_{p}}\left|z_{i}\right|^{2\left(1-\alpha_{i}\right)}$.

We then make use of the distributional identity (3) to get

$$
\begin{aligned}
F\left(c_{1}(L)\right)(v) & =-\int_{X} \hat{\theta}_{v}\left(n \operatorname{Ric}\left(\hat{\omega}_{\infty}\right)-n \mu \hat{\omega}_{\infty}\right) \wedge \frac{\hat{\omega}_{\infty}^{n-1}}{n !} \\
& =-\int_{X} \hat{\theta}_{v}\left[\left(n \operatorname{Ric}(\Omega)-n c_{1}([D], h)-n \mu_{1} \hat{\omega}_{\infty}\right)+n\{D\}-\left(n \mu-n \mu_{1}\right) \hat{\omega}_{\infty}\right] \wedge \frac{\hat{\omega}_{\infty}^{n-1}}{n !} \\
& =-\int_{X} \hat{\theta}_{v}\left(S\left(\hat{\omega}_{\infty}\right)-n \mu_{1}\right) \frac{\hat{\omega}_{\infty}^{n}}{n !}-\int_{X}\{D\} \hat{\theta}_{v} \frac{\hat{\omega}_{\infty}^{n-1}}{(n-1) !}+\left(n \mu-n \mu_{1}\right) \int_{X} \hat{\theta}_{v} \frac{\hat{\omega}_{\infty}^{n}}{n !} \\
& =-\left(\int_{D} \hat{\theta}_{v} \frac{\hat{\omega}_{\infty}^{n-1}}{(n-1) !}-\frac{V o l(D)}{\operatorname{Vol}(X)} \int_{X} \hat{\theta}_{v} \frac{\hat{\omega}_{\infty}^{n}}{n !}\right)
\end{aligned}
$$

So we get

$$
0=F\left(c_{1}(L)\right)(v)+\left(\int_{D} \hat{\theta}_{v} \frac{\hat{\omega}_{\infty}^{n-1}}{(n-1) !}-\frac{\operatorname{Vol}(D)}{\operatorname{Vol}(X)} \int_{X} \hat{\theta}_{v} \frac{\hat{\omega}_{\infty}^{n}}{n !}\right)
$$

Since the two integrals in the above formula is integration of (singular) equivariant forms, they are independent of the chosen Kähler metric in $\overline{\mathcal{P}}(\omega)$ with at worst conic singularities. In particular, we can choose the smooth Kähler metric $\omega$, then we just discover the log-Futaki invariant defined by Donaldson: 
Definition 2. [4]

$$
F\left(c_{1}(L), D\right)(v)=F\left(c_{1}(L)\right)(v)+\left(\int_{D} \theta_{v} \frac{\omega^{n-1}}{(n-1) !}-\frac{\operatorname{Vol}(D)}{\operatorname{Vol}(X)} \int_{X} \theta_{v} \frac{\omega^{n}}{n !}\right)
$$

Remark 2. This differs from the formula in [4] by a sign. And we think of $D$ as a cycle with real coefficients, so if we replace $D$ by $(1-\beta) \triangle$, we have the same formua as that in [4].

\section{3 log-K-energy and Berman's formulation}

We can integrate the log-Futaki-invariant to get log-K-energy

$$
\begin{aligned}
\nu_{\omega, D}(\phi) & =-\int_{0}^{1} d t \int_{X}\left(S\left(\omega_{t}\right)-\underline{S}\right) \dot{\phi} \frac{\omega_{t}^{n}}{n !}+\int_{0}^{1} d t \int_{D} \dot{\phi} \frac{\omega_{t}^{n-1}}{(n-1) !}-\frac{\operatorname{Vol}(D)}{\operatorname{Vol}(X)} \int_{0}^{1} d t \int_{X} \dot{\phi} \frac{\omega_{t}^{n}}{n !} \\
& =\nu_{\omega}(\phi)+\int_{0}^{1} \int_{X}\left(\frac{\sqrt{-1}}{2 \pi} \partial \bar{\partial} \log \|s\|^{2}+c_{1}([D], h)\right) \dot{\phi} \frac{\omega_{t}^{n-1}}{(n-1) !}+\frac{\operatorname{Vol}(D)}{\operatorname{Vol}(X)} F_{\omega}^{0}(\phi) \\
& =\nu_{\omega}(\phi)+\frac{\operatorname{Vol}(D)}{\operatorname{Vol}(X)} F_{\omega}^{0}(\phi)+\mathcal{J}_{\omega}^{\chi_{D}}(\phi)+\int_{X} \log \left\|s_{D}\right\|^{2} \frac{\omega_{\phi}^{n}}{n !}
\end{aligned}
$$

where $\chi_{D}=c_{1}([D], h)$ is the Chern curvature form. The functionals $F_{\omega}^{0}(\phi)$ and $\mathcal{J}_{\omega}^{\chi}(\phi)$ are defined by:

$$
\begin{gathered}
F_{\omega}^{0}(\phi)=-\int_{0}^{1} d t \int_{X} \dot{\phi} \frac{\omega_{\phi_{t}}^{n}}{n !} \\
\mathcal{J}_{\omega}^{\chi}(\phi)=\int_{0}^{1} d t \int_{X} \dot{\phi} \chi \wedge \frac{\omega_{\phi_{t}}^{n-1}}{(n-1) !}
\end{gathered}
$$

Let's now focus on the Fano case as in the beginning of this paper. (2) is equivalent to the following singular complex Monge-Ampère equation:

$$
(\omega+\partial \bar{\partial} \phi)^{n}=e^{-\beta \phi} \frac{\Omega_{1}}{\|s\|^{2(1-\beta)}}
$$

with $\Omega_{1}=e^{h_{\omega}} \omega^{n}$ and $s$ is a defining section of $[Y]$. Note that the line bundle $[Y]=K_{X}^{-1}$ has the Hermitian metric $\|\cdot\|$ such that the curvature is $\omega$.

We have $D=(1-\beta) Y$. Since $[Y]=K_{X}^{-1}$, we can assume $\chi_{D}=(1-\beta) \omega, \operatorname{Vol}((1-\beta) D)=$ $n(1-\beta) \operatorname{Vol}(X)$. Then $(7)$ becomes

$$
\begin{aligned}
\nu_{\omega, D} & =\nu_{\omega}(\phi)+(1-\beta)\left(n F_{\omega}^{0}(\phi)+\mathcal{J}_{\omega}^{\omega}(\phi)\right)+(1-\beta) \int_{X} \log \|s\|^{2} \frac{\omega_{\phi}^{n}}{n !} \\
& =\nu_{\omega}(\phi)+(1-\beta)\left(I_{\omega}-J_{\omega}\right)+(1-\beta) \int_{X} \log \|s\|^{2} \frac{\omega_{\phi}^{n}}{n !} \\
& =\int_{X} \log \frac{\omega_{\phi}^{n}}{\Omega_{1}}-\beta\left(I_{\omega}-J_{\omega}\right)+(1-\beta) \int_{X} \log \|s\|^{2} \frac{\omega_{\phi}^{n}}{n !}
\end{aligned}
$$

We have used the well known formula for K-energy [12]:

$$
\nu_{\omega}(\phi)=\int_{X} \log \frac{\omega_{\phi}^{n}}{\Omega_{1}}-\left(I_{\omega}-J_{\omega}\right)(\phi)
$$

where

$$
I_{\omega}(\phi)=\int_{X} \phi\left(\omega^{n}-\omega_{\phi}^{n}\right) / n !
$$




$$
J_{\omega}(\phi)=F_{\omega}^{0}(\phi)+\int_{X} \phi \frac{\omega^{n}}{n !}
$$

And it's easy to verify that

$$
n F_{\omega}^{0}(\phi)+\mathcal{J}_{\omega}^{\omega}(\phi)=\left(I_{\omega}-J_{\omega}\right)(\phi)=-\left(\int_{X} \phi \omega_{\phi}^{n}+F_{\omega}^{0}(\phi)\right)
$$

From above formula, we see that, in Fano case, the log-K-energy coincides with Berman's free energy associated with (8) ([1])

$$
\nu_{\omega, D}=\int_{X} \log \frac{\omega_{\phi}^{n}}{\Omega_{1} /\|s\|^{2(1-\beta)}} \frac{\omega_{\phi}^{n}}{n !}+\beta\left(\int_{X} \phi \omega_{\phi}^{n}+F_{\omega}^{0}(\phi)\right)
$$

\section{Log-K-stability}

We imitate the definition of K-stability to define log-K-stability. First we recall the definition of test configuration [3] or special degeneration [14] of a polarized projective variety $(X, L)$.

Definition 3. A test configuration of $(X, L)$, consists of

1. a scheme $\mathcal{X}$ with $a \mathbb{C}^{*}$-action;

2. $a \mathbb{C}^{*}$-equivariant line bundle $\mathcal{L} \rightarrow \mathcal{X}$

3. a flat $\mathbb{C}^{*}$-equivariant map $\pi: \mathcal{X} \rightarrow \mathcal{C}$, where $\mathbb{C}^{*}$ acts on $\mathbb{C}$ by multiplication in the standard way;

such that any fibre $X_{t}=\pi^{-1}(t)$ for $t \neq 0$ is isomorphic to $X$ and $(X, L)$ is isomorphic to $\left(X_{t},\left.\mathcal{L}\right|_{X_{t}}\right)$.

Any test configuration can be equivariantly embedded into $\mathbb{P}^{N} \times \mathbb{C}^{*}$ where the $\mathbb{C}^{*}$ action on $\mathbb{P}^{N}$ is given by a 1 parameter subgroup of $S L(N+1, \mathbb{C})$. If $Y$ is any subvariety of $X$, the test configuration of $(X, L)$ also induces a test configuration $\left(\mathcal{Y},\left.\mathcal{L}\right|_{\mathcal{Y}}\right)$ of $\left(Y,\left.L\right|_{Y}\right)$.

Let $d_{k}, \tilde{d}_{k}$ be the dimensions of $H^{0}\left(X, L^{k}\right), H^{0}\left(Y,\left.L\right|_{Y} ^{k}\right)$, and $w_{k}, \tilde{w}_{k}$ be the weights of $\mathbb{C}^{*}$ action on $H^{0}\left(X_{0},\left.\mathcal{L}\right|_{X_{0}} ^{k}\right), H^{0}\left(Y_{0},\left.\mathcal{L}\right|_{Y_{0}} ^{k}\right)$, respectively. Then we have expansions:

$$
\begin{gathered}
w_{k}=a_{0} k^{n+1}+a_{1} k^{n}+O\left(k^{n-1}\right), \quad d_{k}=b_{0} k^{n}+b_{1} k^{n-1}+O\left(k^{n-2}\right) \\
\tilde{w}_{k}=\tilde{a}_{0} k^{n}+O\left(k^{n-1}\right), \quad \tilde{d}_{k}=\tilde{b}_{0} k^{n-1}+O\left(k^{n-2}\right)
\end{gathered}
$$

If the central fibre $X_{0}$ is smooth, we can use equivariant differential forms to calculate the coefficients by [3]. Let $\omega$ be a smooth Kähler form in $c_{1}(L)$, and $\theta_{v}=\mathcal{L}_{v}-\nabla_{v}$, then

$$
\begin{gathered}
a_{0}=-\int_{X} \theta_{v} \frac{\omega^{n}}{n !} ; a_{1}=-\frac{1}{2} \int_{X} \theta_{v} S(\omega) \frac{\omega^{n}}{n !} \\
b_{0}=\int_{X} \frac{\omega^{n}}{n !}=\operatorname{Vol}(X) ; b_{1}=\frac{1}{2} \int_{X} S(\omega) \frac{\omega^{n}}{n !} \\
\tilde{a}_{0}=-\int_{Y_{0}} \theta_{v} \frac{\omega^{n-1}}{(n-1) !} ; \tilde{b}_{0}=\int_{Y_{0}} \frac{\omega^{n-1}}{(n-1) !}=\operatorname{Vol}\left(Y_{0}\right)
\end{gathered}
$$

Remark 3. To see the signs of coefficients and give an example, we consider the case where $X=\mathbb{P}^{1}, L=\mathcal{O}_{\mathbb{P}^{1}}(k)$. $\mathbb{C}^{*}$ acts on $\mathbb{P}^{1}$ by multiplication: $t \cdot z=t z$. A general $D \in|L|$ consists of $k$ points. As $t \rightarrow 0, t \cdot D \rightarrow k\{0\} . D$ is the zero set of a general degree $k$ homogeneous polynomial $P_{k}\left(z_{0}, z_{1}\right)$ and $k\{0\}$ is the zero set of $z_{1}^{k}$. $\mathbb{C}^{*}$ acts on $H^{0}\left(\mathbb{P}^{1}, \mathcal{O}(k)\right)$ by $t \cdot z_{0}^{i} z_{1}^{j}=t^{-j} z_{0}^{i} z_{1}^{j}$ 
so that $\lim _{t \rightarrow 0}\left[t \cdot P_{k}\left(z_{0}, z_{1}\right)\right]=\left[z_{1}^{k}\right]$, where $\left[P_{k}\right] \in \mathbb{P}\left(H^{0}\left(\mathbb{P}^{1}, \mathcal{O}(k)\right)\right)$. Take the Fubini-Study metric $\omega_{F S}=\frac{\sqrt{-1}}{2 \pi} \partial \bar{\partial} \log \left(1+|z|^{2}\right)=\frac{\sqrt{-1}}{2 \pi} \frac{d z \wedge d \bar{z}}{\left(1+|z|^{2}\right)^{2}}$, then $\theta_{v}=\frac{\partial \log \left(1+|z|^{2}\right)}{\partial \log |z|^{2}}=\frac{|z|^{2}}{1+|z|^{2}}$. So

$$
\begin{aligned}
& -a_{0}=\int_{\mathbb{P}^{1}} \theta_{v} \omega_{F S}=\int_{0}^{+\infty} \frac{r^{2}}{\left(1+r^{2}\right)^{3}} 2 r d r=\frac{1}{2} \\
& -a_{1}=\frac{1}{2} \int_{\mathbb{P}^{1}} S\left(\omega_{F S}\right) \theta_{v} \omega_{F S}=\int_{\mathbb{P}^{1}} \theta_{v} \omega_{F S}=\frac{1}{2}
\end{aligned}
$$

While

$$
w_{k}=-(1+\cdots+k)=-\frac{1}{2} k^{2}-\frac{1}{2} k
$$

which gives exactly $a_{0}=a_{1}=-\frac{1}{2}$.

Comparing (6), (9)-(11), we can define the algebraic log-Futaki invariant of the given test configuration to be

$$
\begin{aligned}
F(\mathcal{X}, \mathcal{Y}, \mathcal{L}) & =\frac{2\left(a_{1} b_{0}-a_{0} b_{1}\right)}{b_{0}}+\left(-\tilde{a}_{0}+\frac{\tilde{b}_{0}}{b_{0}} a_{0}\right) \\
& =\frac{\left(2 a_{1}-\tilde{a}_{0}\right) b_{0}-a_{0}\left(2 b_{1}-\tilde{b}_{0}\right)}{b_{0}}
\end{aligned}
$$

Definition 4. $(X, Y, L)$ is log-K-stable along the test configuration $(\mathcal{X}, \mathcal{L})$ if $F(\mathcal{X}, \mathcal{Y}, \mathcal{L}) \leq 0$, and equality holds if and only if $(\mathcal{X}, \mathcal{Y}, \mathcal{L})$ is a product configuration.

$(X, Y, L)$ is semi-log-K-stable along $(\mathcal{X}, \mathcal{L})$ if $F(\mathcal{X}, \mathcal{Y}, \mathcal{L}) \leq 0$. Otherwise, it's unstable.

$(X, Y, L)$ is log-K-stable (semi-log-K-stable) if, for any integer $r>0,\left(X, Y, L^{r}\right)$ is log-K-stable (semi-log-K-stable) along any test configuration of $\left(X, Y, L^{r}\right)$.

Remark 4. When $Y$ is empty, then definition of log-K-stability becomes the definition of $K$ stability. ([14], [3])

Remark 5. In applications, we sometimes meet the following situation. Let $\lambda(t): \mathbb{C}^{*} \rightarrow S L(N+$ $1, \mathbb{C})$ be a 1 parameter subgroup. As $t \rightarrow \infty, \lambda(t)$ will move $X, Y \subset \mathbb{P}^{N}$ to the limit scheme $X_{0}, Y_{0}$. Then stability condition is equivalent to the other opposite sign condition $F\left(X_{0}, Y_{0}, v\right) \geq 0$. This is of course related to the above definition by transformation $t \rightarrow t^{-1}$.

Example 1 (Orbifold). Assume $X$ is smooth. $Y=\sum_{i=1}^{r}\left(1-\frac{1}{n_{i}}\right) D_{i}$ is a normal crossing divisor, where $n_{i}>0$ are integers. The conic Kähler metric on $(X, Y)$ is just the orbifold Kähler metric on the orbifold $(X, Y)$. Orbifold behaves similarly as smooth variety, but in the calculation, we need to use orbifold canonical bundle $K_{\text {orb }}=K_{X}+Y$. For example, think $L$ as an orbifold line bundle on $X$, then the orbifold Riemann-Roch says that

$$
\begin{aligned}
\operatorname{dim} H_{\text {orb }}^{0}((X, Y), L) & =\frac{L^{n}}{n !} k^{n}+\frac{1}{2} \frac{-\left(K_{X}+Y\right) \cdot L^{n}}{(n-1) !} k^{n-1}+O\left(k^{n-2}\right) \\
& =b_{0} k^{n}+\frac{1}{2}\left(2 b_{1}-\tilde{b}_{0}\right) k^{n-1}+O\left(k^{n-2}\right)
\end{aligned}
$$

For the $\mathbb{C}^{*}$-weight of $H_{\text {orb }}^{0}((X, Y), L)$, we have expansion:

$$
w_{k}^{o r b}=a_{0}^{o r b} k^{n+1}+a_{1}^{o r b} k^{n}+O\left(k^{n-1}\right)
$$

By orbifold equivariant Riemann-Roch, we have the formula:

$$
a_{0}^{\text {orb }}=\int_{X} \hat{\theta}_{v} \frac{\hat{\omega}^{n}}{n !}=\int_{X} \theta_{v} \frac{\omega^{n}}{n !}=a_{0}
$$




$$
a_{1}^{o r b}=\int_{X} \hat{\theta}_{v} S(\hat{\omega}) \frac{\hat{\omega}^{n}}{n !}
$$

To calculate the second coefficient $a_{1}^{\text {orb }}$, we choose an orbifold metric $\hat{\omega}$, then by (9):

$$
\begin{aligned}
a_{1} & =-\frac{1}{2} \int_{X} \hat{\theta}_{v} n \operatorname{Ric}(\hat{\omega}) \wedge \frac{\hat{\omega}^{n-1}}{n !} \\
& =-\frac{1}{2} \int_{X} \hat{\theta}_{v} n\left(\operatorname{Ric}(\Omega)-c_{1}([D], h)+\{D\}\right) \wedge \frac{\hat{\omega}^{n-1}}{n !} \\
& =-\frac{1}{2} \int_{X} \hat{\theta}_{v} S(\hat{\omega}) \frac{\hat{\omega}^{n}}{n !}-\frac{1}{2} \int_{D} \hat{\theta}_{v} \frac{\hat{\omega}^{n-1}}{(n-1) !} \\
& =a_{1}^{\text {orb }}-\frac{1}{2} \int_{D} \theta_{v} \frac{\omega^{n-1}}{(n-1 !}=a_{1}^{\text {orb }}+\frac{1}{2} \tilde{a}_{0}
\end{aligned}
$$

So

$$
a_{1}^{\text {orb }}=\frac{1}{2}\left(2 a_{1}-\tilde{a}_{0}\right)
$$

Comparing (12), we see that the log-Futaki invariant recovers the orbifold Futaki invariant, and similarly log-K-stability recovers orbifold K-stability. Orbifold Futaki and orbifold K-stability were studied by Ross-Thomas [9].

Example 2. $X=\mathbb{P}^{1}, L=K_{\mathbb{P}^{1}}^{-1}=\mathcal{O}_{\mathbb{P}^{1}}(2), Y=\sum_{i=1}^{r} \alpha_{i} p_{i}$. For any $i \in\{1, \cdots, r\}$, we choose the coordinate $z$ on $\mathbb{P}^{1}$, such that $z\left(p_{i}\right)=0$. Then consider the holomorphic vector field $v=z \partial_{z}$. $v$ generates the 1 parameter subgroup $\lambda(t): \lambda(t) \cdot z=t \cdot z$. As $t \rightarrow \infty, \lambda(t)$ degenerate $(X, Y)$ into the pair $\left(\mathbb{P}^{1}, \alpha_{i}\{0\}+\sum_{j \neq i} \alpha_{j}\{\infty\}\right)$. We take $\theta_{v}=\frac{-|z|^{-2}+|z|^{2}}{|z|^{-2}+1+|z|^{2}}$. Then it's easy to get the log-Futaki invariant of the degeneration determined by $\lambda$ :

$$
F\left(\mathbb{P}^{1}, \sum_{i=1}^{r} \alpha_{i} p_{i}, \mathcal{O}_{\mathbb{P}^{1}}(2)\right)(\lambda)=\sum_{j \neq i} \alpha_{j}-\alpha_{i}
$$

If $\left(\mathbb{P}^{1}, \sum_{i=1}^{r} \alpha_{i} p_{i}\right)$ is log-K-stable, by Remark 5 , we have

$$
\sum_{j \neq i} \alpha_{j}-\alpha_{i}>0
$$

Equivalently, if we let $t \rightarrow 0$, we get $\alpha_{i}-\sum_{j \neq i} \alpha_{j}<0$ from log-K-stability.

Let's consider the problem of constructing singular Riemannian metric $g$ of constant scalar curvature on $\mathbb{P}^{1}$ which has conic angle $2 \pi\left(1-\alpha_{i}\right)$ at $p_{i}$ and is smooth elsewhere. Assume $p_{i} \neq \infty$ for any $i=1, \ldots, r$. Under conformal coordinate $z$ of $\mathbb{C} \subset \mathbb{P}^{1}, g=e^{2 u}|d z|^{2}$. u is a smooth function in the punctured complex plane $\mathbb{C}-\left\{p_{1}, \ldots, p_{r}\right\}$ so that near each $p_{i}, u(z)=-2 \alpha_{i} \log \left|z-p_{i}\right|+a$ continuous function, where $\alpha_{i} \in(0,1)$ and $u=-2 \log |z|+$ a continuous function near infinity. We call such function is of conic type. The condition of constant scalar curvature corresponds to the following Liouville equations.

1. $\Delta u=-e^{2 u}$

2. $\Delta u=0$

3. $\Delta u=e^{2 u}$

which correspond to scalar curvature $=1,0,-1$ case respectively.

For such equations, we have the following nice theorem due to Troyanov, McOwen, Thurston, Luo-Tian. 
Theorem 3 (See [7] and the reference there). 1. For equation 1, it has a solution of conic type if and only if

(a) $\sum_{i=1}^{r} \alpha_{i}<2$, and

(b) $\sum_{j \neq i} \alpha_{j}-\alpha_{i}>0$, for all $i=1, \ldots, n$.

2. For equation 2, it has a solution of conic type if and only if $(a): \sum_{i=1}^{r} \alpha_{i}=2$.

In this case, (a) implies the condition: (b) $\sum_{j \neq i} \alpha_{j}-\alpha_{i}>0$, for all $i=1, \ldots, r$.

3. For equation 3, it has a solution of conic type if and only if $(a): \sum_{i=1}^{r} \alpha_{i}>2$.

Again in this case, (a) implies the condition: (b) $\sum_{j \neq i} \alpha_{j}-\alpha_{i}>0$, for all $i=1, \ldots, r$.

Moreover, the above solutions are all unique.

Note that $\operatorname{deg}\left(-\left(K_{\mathbb{P}^{1}}+\sum_{i=1}^{r} \alpha_{i} p_{i}\right)\right)=2-\sum_{i=1}^{r} \alpha_{i}$, so by (4), conditions (a) in above theorem correspond to the cohomological conditions for the scalar curvature to be positive, zero, negative respectively. While the condition (b) is the same as (14). So by the above theorem, if $\left(\mathbb{P}^{1}, \sum_{i=1}^{r} \alpha_{i} p_{i}\right)$ is log-K-stable, then there is a conic metric on $\left(\mathbb{P}^{1}, \sum_{i=1}^{r} \alpha_{i} p_{i}\right)$ with constant curvature whose sign is the same as that of $2-\sum_{i} \alpha_{i}$.

This example clearly suggests

Conjecture 3 (Logarithmic version of Tian-Yau-Donaldson conjecture). There is a constant scalar curvature conic Kähler metric on $(X, Y)$ if and only if $(X, Y)$ is log-K-stable.

\section{Toric Fano case}

\subsection{Log-Futaki invariant for 1psg on toric Fano variety}

For a reflexive lattice polytope $\triangle$ in $\mathbb{R}^{n}=\Lambda \otimes_{\mathbb{Z}} \mathbb{R}$, we have a Fano toric manifold $\left(\mathbb{C}^{*}\right)^{n} \subset X_{\triangle}$ with a $\left(\mathbb{C}^{*}\right)^{n}$ action. In the following, we will sometimes just write $X$ for $X_{\triangle}$ for simplicity.

Let $\left(S^{1}\right)^{n} \subset\left(\mathbb{C}^{*}\right)^{n}$ be the standard real maximal torus. Let $\left\{z_{i}\right\}$ be the standard coordinates of the dense orbit $\left(\mathbb{C}^{*}\right)^{n}$, and $x_{i}=\log \left|z_{i}\right|^{2}$. We have

Lemma 1. Any $\left(S^{1}\right)^{n}$ invariant Kähler metric $\omega$ on $X$ has a potential $u=u(x)$ on $\left(\mathbb{C}^{*}\right)^{n}$, i.e. $\omega=\frac{\sqrt{-1}}{2 \pi} \partial \bar{\partial} u$. $u$ is a proper convex function on $\mathbb{R}^{n}$, and satisfies the momentum map condition:

$$
D u\left(\mathbb{R}^{n}\right)=\triangle
$$

Also,

$$
\frac{(\partial \bar{\partial} u)^{n} / n !}{\frac{d z_{1}}{z_{1}} \wedge \frac{d \bar{z}_{1}}{\bar{z}_{1}} \cdots \wedge \frac{d z_{n}}{z_{n}} \wedge \frac{d \bar{z}_{n}}{\bar{z}_{n}}}=\operatorname{det}\left(\frac{\partial^{2} u}{\partial x_{i} \partial x_{j}}\right)
$$

Let $\left\{p_{\alpha} ; \alpha=1, \cdots, N\right\}$ be all the lattice points of $\triangle$. Each $p_{\alpha}$ corresponds to a holomorphic section $s_{\alpha} \in H^{0}\left(X_{\triangle}, K_{X_{\triangle}}^{-1}\right)$. We can embed $X_{\triangle}$ into $\mathbb{P}^{N}$ using $\left\{s_{\alpha}\right\}$. Define $u$ to be the potential on $\left(\mathbb{C}^{*}\right)^{n}$ for the pull back of Fubini-Study metric (i.e. $\frac{\sqrt{-1}}{2 \pi} \partial \bar{\partial} u=\omega_{F S}$ ):

$$
u=\log \left(\sum_{\alpha=1}^{N} e^{<p_{\alpha}, x>}\right)+C
$$

$C$ is some constant determined by normalization condition:

$$
\int_{\mathbb{R}^{n}} e^{-u} d x=\operatorname{Vol}(\triangle)=\frac{1}{n !} \int_{X_{\triangle}} \omega^{n}=\frac{c_{1}\left(X_{\triangle}\right)^{n}}{n !}
$$


By the above normalization of $u$, it's easy to see that

$$
e^{h_{\omega}}=\frac{|\cdot|_{F S}^{2}}{|\cdot|_{\omega^{n}}^{2}}=\frac{e^{-u}}{\omega^{n} /\left(\frac{d z_{1}}{z_{1}} \wedge \frac{d \bar{z}_{1}}{\bar{z}_{1}} \cdots \wedge \frac{d z_{n}}{z_{n}} \wedge \frac{\left.d \bar{z}_{n}\right)}{\bar{z}_{n}}\right.}
$$

So

$$
h_{\omega}=-\log \operatorname{det}\left(u_{i j}\right)-u
$$

Now let's calculate the log-Futaki invariant for any 1-parameter subgroup in $\left(\mathbb{C}^{*}\right)^{n}$. Each 1parameter subgroup in $\left(\mathbb{C}^{*}\right)^{n}$ is determined by some $\lambda \in \mathbb{R}^{n}$ such that the generating holomorphic vector field is

$$
v_{\lambda}=\sum_{i=1}^{n} \lambda_{i} z_{i} \frac{\partial}{\partial z_{i}}
$$

A general Calabi-Yau hypersurface $Y \in\left|-K_{X}\right|$ is a hyperplane section given by the equation:

$$
s:=\sum_{\alpha=1}^{N} b\left(p_{\alpha}\right) z^{p_{\alpha}}=0
$$

By abuse of notation, we denote $\lambda(t)$ to be the 1 parameter subgroup generated by $v_{\lambda}$, then

$$
\lambda(t) \cdot s=\sum_{\alpha=1}^{N} b\left(p_{\alpha}\right) t^{-\left\langle p_{\alpha}, \lambda\right\rangle} z^{p_{\alpha}}
$$

Let

$$
W(\lambda)=\max _{p \in \Delta}\langle p, \lambda\rangle
$$

Then $H_{\lambda}=\left\{p \in \mathbb{R}^{n},\langle p, \lambda\rangle=W(\lambda)\right\}$ is a supporting plane of $\triangle$, and

$$
\mathcal{F}_{\lambda}:=\{p \in \triangle ;\langle p, \lambda\rangle=W(\lambda)\}=H_{\lambda} \cap \triangle
$$

is a face of $\triangle$.

We have $\lim _{t \rightarrow 0}[s]=\left[s_{0}:=\sum_{p_{\alpha} \in \mathcal{F}_{\lambda}} b\left(p_{\alpha}\right) z^{p_{\alpha}}\right]$, and by (18), the $C^{*}$-weight of $s_{0}$ is $-W(\lambda)$.

Proposition 2. Let $F\left(K_{X}^{-1}, \beta Y\right)(\lambda)$ denote the Futaki invariant of the test configuration associated with the 1 parameter subgroup generated by $v_{\lambda}$. We have

$$
F\left(K_{X}^{-1}, \beta Y\right)(\lambda)=-\left(\beta\left\langle P_{c}, \lambda\right\rangle+(1-\beta) W(\lambda)\right) \operatorname{Vol}(\triangle)
$$

Proof. We will use the algebraic definition of log-Futaki invariant (12) to do the calculation.

Note that $\left(X, Y, K_{X}^{-1}\right)$ degenerates to $\left(X, Y_{0}, K_{X}^{-1}\right)$ under $\lambda$.

$Y_{0}$ is a hyperplane section of $X$, and $s_{0} \in H^{0}\left(X, K_{X}^{-1}\right)$ is the defining section, i.e. $Y_{0}=\left\{s_{0}=0\right\}$. Then

$$
H^{0}\left(Y_{0},\left.K_{X}^{-1}\right|_{Y_{0}} ^{k}\right) \cong H^{0}\left(X, K_{X}^{-k}\right) /\left(s_{0} \otimes H^{0}\left(X, K_{X}^{-(k-1)}\right)\right)
$$

So

$$
\tilde{w}_{k}=w_{k}-\left(w_{k-1}-W(\lambda) d_{k-1}\right)
$$

Plugging the expansions, we get

$$
\tilde{a}_{0}=(n+1) a_{0}+W(\lambda) b_{0}
$$

Note that $\tilde{b}_{0}=n b_{0}=n \operatorname{Vol}(\triangle)$, we have

$$
-\tilde{a}_{0}+\frac{\tilde{b}_{0}}{b_{0}} a_{0}=-a_{0}-W(\lambda) b_{0}
$$


where

$$
-a_{0}=\int_{X} \theta_{v} \frac{\omega^{n}}{n !}=\int_{\mathbb{R}^{n}} \sum_{i} \lambda_{i} u_{i} \operatorname{det}\left(u_{i j}\right) d x=\int_{\triangle} \sum_{i} \lambda_{i} y_{i} d y=\operatorname{Vol}(\triangle)\left\langle P_{c}, \lambda\right\rangle
$$

By (17), the ordinary Futaki invariant is given by

$$
\begin{aligned}
F\left(c_{1}(X)\right)\left(v_{\lambda}\right) & =\int_{X} v\left(h_{\omega}\right) \frac{\omega^{n}}{n !}=-\int_{\mathbb{R}^{n}} \sum_{i=1}^{n} \lambda_{i} \frac{\partial u}{\partial x_{i}} \operatorname{det}\left(u_{i j}\right) d x \\
& =-\int_{\triangle} \sum_{i} \lambda_{i} y_{i} d y=-\operatorname{Vol}(\triangle)\left\langle P_{c}, \lambda\right\rangle
\end{aligned}
$$

Substituting these into (12), we get

$$
\begin{aligned}
F\left(K_{X}^{-1}, \beta Y\right)(\lambda) & =-\operatorname{Vol}(\triangle)\left\langle P_{c}, \lambda\right\rangle+(1-\beta)\left(\operatorname{Vol}(\triangle)\left\langle P_{c}, \lambda\right\rangle-W(\lambda) \operatorname{Vol}(\triangle)\right) \\
& =-\left(\beta\left\langle P_{c}, \lambda\right\rangle+(1-\beta) W(\lambda)\right) \operatorname{Vol}(\triangle)
\end{aligned}
$$

Proof of Theorem 1. Note that for any $P_{\lambda} \in \mathcal{F}_{\lambda} \subset \partial \triangle, W(\lambda)=\left\langle P_{\lambda}, \lambda\right\rangle$. By Theorem 2, we have

$$
\begin{aligned}
F\left(K_{X}^{-1}, \beta Y\right)(\lambda) & =\left(\frac{\beta}{1-\beta} \frac{1-R(X)}{R(X)}\langle Q, \lambda\rangle-W(\lambda)\right)(1-\beta) \operatorname{Vol}(\triangle) \\
& =\left\langle Q_{\beta}-P_{\lambda}, \lambda\right\rangle
\end{aligned}
$$

where $Q_{\beta}=\frac{\beta}{1-\beta} \frac{1-R(X)}{R(X)} Q$.

Note that $\lambda$ is a outward normal vector of $H_{\lambda}$. By convexity of $\triangle$, it's easy to see that (see the picture after Example 2)

- $\beta<R(X): Q_{\beta} \in \triangle^{\circ}$. For any $\lambda \in \mathbb{R}^{n},\left\langle Q_{\beta}-P_{\lambda}, \lambda\right\rangle<0$.

- $\beta=R(X): Q_{\beta}=Q \in \partial \triangle$. For any $\lambda \in \mathbb{R}^{n},\left\langle Q_{\beta}-P_{\lambda}, \lambda\right\rangle \leq 0$. Equality holds if and only if $\langle Q, \lambda\rangle=W(\lambda)$, i.e. $H_{\lambda}$ is a supporting plane of $\triangle$ at point $Q$.

- $\beta>R(X): Q_{\beta} \notin \bar{\triangle}$. There exists $\lambda \in \mathbb{R}^{n}$ such that $\left\langle Q_{\beta}-P_{\lambda}, \lambda\right\rangle>0$

\subsection{Example}

1. $X_{\triangle}=B l_{p} \mathbb{P}^{2}$. See the picture in Introduction. $P_{c}=\frac{1}{4}\left(\frac{1}{3}, \frac{1}{3}\right), Q=-6 P_{c} \in \partial \triangle$, so $R(X)=\frac{6}{7}$. If we take $\lambda=\langle-1,-1\rangle$, then $W(\lambda)=1$. So by (19)

$$
F\left(K_{X}^{-1}, \beta Y\right)(\lambda)=\frac{2}{3} \beta-4(1-\beta)
$$

So $F\left(K_{X}^{-1}, \beta Y\right)(\lambda) \leq 0$ if and only if $\beta \leq \frac{6}{7}$, and equality holds exactly when $\beta=\frac{6}{7}$.

2. $X_{\triangle}=B l_{p, q} \mathbb{P}^{2}, P_{c}=\frac{2}{7}\left(-\frac{1}{3},-\frac{1}{3}\right), Q=-\frac{21}{4} P_{c} \in \partial \triangle$, so $R\left(X_{\triangle}\right)=\frac{21}{25}$.

If we take $\lambda_{1}=\langle 1,1\rangle$, then $W\left(\lambda_{1}\right)=1$. By (19),

$$
F\left(K_{X}^{-1}, \beta Y\right)\left(\lambda_{1}\right)=\frac{2}{3} \beta-\frac{7}{2}(1-\beta)
$$

$F\left(K_{X}^{-1}, \beta Y\right)\left(\lambda_{1}\right) \leq 0$ if and only if $\beta \leq \frac{21}{25}$.

This is essentially the same as Donaldson's calculation in [4]. 
If we take $\lambda_{3}=\langle-1,2\rangle$, then $W\left(\lambda_{3}\right)=\langle-1,2\rangle \cdot\langle-1,1\rangle=3$. By (19)

$$
F\left(K_{X}^{-1}, \beta Y\right)\left(\lambda_{3}\right)=\frac{1}{3} \beta-\frac{21}{2}(1-\beta)
$$

So $F\left(K_{X}^{-1}, \beta Y\right)\left(\lambda_{3}\right) \leq 0$ if and only if $\beta \leq \frac{63}{65}$ which means that $(X, \beta Y)$ is log-K-stable along $\lambda_{3}$ when $\beta \leq \frac{21}{25}<\frac{63}{65}$.

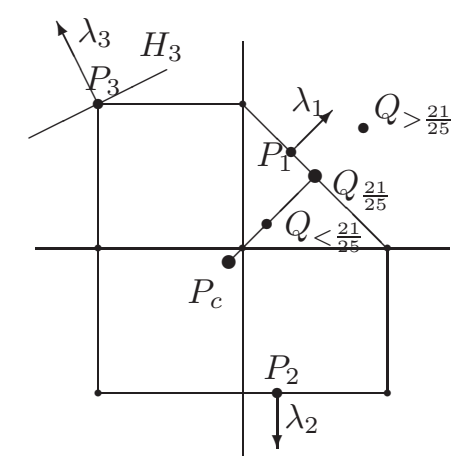

\section{References}

[1] Berman, R.: A thermodynamic formalism for Monge-Ampère euations, Moser-Trudinger inequalities and Kähler-Einstein metrics arXiv:1011.3976

[2] Calabi, E.: Extremal Kähler metrics II, in "Differential geometry and complex analysis", Springer-Verlag, Berlin, Heidelberg, New York, 1985, pp. 95-114.

[3] Donaldson, S.K.: Scalar curvature and stability of toric varieties, Jour. Differential Geometry 62 289-349 (2002)

[4] Donaldson, S.K.: Kähler metrics with cone singularities along a divisor, arXiv:1102.1196

[5] Futaki, A.: An obstruction to the existence of Einstein Kähler metrics, Invent. Math., 73, 437-443 (1983)

[6] Li, C.: Greatest lower bounds on Ricci curvature for toric Fano manifolds, arXiv:0909.3443

[7] Luo, F. and Tian, G.: Liouville equation and spherical convex polytopes, Proceedings of the American Mathematical Society, Vol 116, No. 4 (1992) 1119-1129

[8] Oda, T.: Convex bodies and algebraic geometry-an introduction to the theory of toric varieties, Springer-Vergla, 1988

[9] Ross, J., and Thomas, R.: Weighted projective embeddings, stability of orbifolds and constant scalar curvature Kähler metrics arXiv:0907.5214

[10] Székelyhidi, G.: Greatest lower bounds on the Ricci curvature of Fano manifolds, arXiv:0903.5504

[11] Tian, G.: Kähler-Einstein metrics on algebraic manifolds. Lecture notes in Mathematics, 1996, Volume 1646/1996, 143-185. 
[12] Tian, G.: Canonical Metrics on Kähler Manifolds, Birkhauser, 1999

[13] Tian, G.: On stability of the tangent bundles of Fano varieties. Internat. J. Math. 3, 3(1992), 401-413

[14] Tian, G.: Kähler-Einstein metrics with positive scalar curvature, Invent. Math. 130 (1997), $1-39$

[15] Wang, X.J. and Zhu, X.H.: Kähler-Ricci solitons on toric manifolds with positive first Chern class. Advances in Math. 188 (2004) 87-103

[16] Yau, S.T.: On the Ricci curvature of a compact Kähler manifold and the complex MongeAmpère equation, I, Comm. Pure Appl. Math. 31 (1978) 339-441.

Department of Mathematics, Princeton University, Princeton, NJ 08544, USA

E-mail address: chil@math.princeton.edu 